Electrostatic Potentials and Fields in the Vicinity of Engineered Nanostructures

C.M. Schaldach, W.L. Bourcier, P.H. Paul, W.D. Wilson

March 18, 2004 
This document was prepared as an account of work sponsored by an agency of the United States Government. Neither the United States Government nor the University of California nor any of their employees, makes any warranty, express or implied, or assumes any legal liability or responsibility for the accuracy, completeness, or usefulness of any information, apparatus, product, or process disclosed, or represents that its use would not infringe privately owned rights. Reference herein to any specific commercial product, process, or service by trade name, trademark, manufacturer, or otherwise, does not necessarily constitute or imply its endorsement, recommendation, or favoring by the United States Government or the University of California. The views and opinions of authors expressed herein do not necessarily state or reflect those of the United States Government or the University of California, and shall not be used for advertising or product endorsement purposes.

This work was performed under the auspices of the U.S. Department of Energy by University of California, Lawrence Livermore National Laboratory under Contract W-7405-Eng-48. 


\title{
Electrostatic potentials and fields in the vicinity of engineered nanostructures
}

\author{
C. M. Schaldach ${ }^{1}$, William L. Bourcier ${ }^{1}$, Phillip H. Paul ${ }^{2}$ and W. D. Wilson ${ }^{1 \#}$ \\ ${ }^{1}$ Lawrence Livermore National Laboratory \\ Livermore, CA 94550 \\ ${ }^{2}$ Eksigent Technologies \\ Livermore, CA 94550 \\ ${ }^{\#}$ To whom correspondence should be sent: \\ Lawrence Livermore National Laboratory \\ 7000 East Avenue, L-232 \\ Livermore, CA 94550 \\ (925) 424-2497 \\ wdwils@1ln1.gov
}




\begin{abstract}
We have developed a method of calculation of the electrostatic potentials and fields in the vicinity of geometrically complex engineered nanostructures comprised of varying materials in electrolytes of arbitrary $\mathrm{pH}$ and ionic strength. The method involves direct summation of charged Debye-Hückel spheres comprising the nanostructural surfaces and, by including charge redistribution on the surface of conducting materials held at constant potential, is applicable to mixed boundary conditions. The method is validated by comparison to analytical solutions for an infinite plane (Gouy-Chapman), an infinite cylinder (Bessel functions) and an infinite plane which contains a hole and which is held at constant potential. Excellent agreement between the potentials obtained by our numerical method and the closed form solutions is found for these conditions. The method is applied to the calculation of the electric field enhancement in the vicinity of a nanomembrane whose pore wall is held at constant charge and whose membrane surfaces are held at constant potential. The electric field is found to be enhanced by the charge buildup in the rim of the hole of the nanomembrane, which redistribution results from the potential being held constant in the conducting region. Ion concentrations are also calculated; positive ion rejection is found to be enhanced by this charge buildup in the region of the rim when a constant positive potential is applied.
\end{abstract}

Keywords: Nanotechnology, membranes, Poisson-Boltzmann, electrostatic potentials

\title{
I. Introduction
}

Nanostructural engineering applied to membranes may lead to breakthrough electrochemical separation technologies for the removal of toxins such as nitrates, dioxins 
and perchlorates from contaminated water [1] as well as desalinization of brackish and sea water [2]. Computational approaches capable of handling complex geometries, materials and applied fields may lead to design rules for these membranes which are difficult to discover by direct experimentation alone. As a first step toward that goal, we have developed methods to calculate the electrostatic potentials and fields in the vicinity of geometrically complex nanostructures, selective membranes, comprised of varying materials in electrolytes of varying $\mathrm{pH}$ and ionic strength.

Selective membranes are well known in biology, where ion channels form a selectivity filter. Tieleman, et al [3], are designing an ion channel to be anion selective by mutation of pore-lining amino acid residues, from glutamines to lysines. These authors have investigated the $\mathrm{pH}$-dependence and channel stability using finite element-based PoissonBoltzmann and molecular dynamics calculations. Miedema [4] has shown how ion channel surface potentials may be responsible for a reversal of the calculated permeability ratio, $\mathrm{P}_{\mathrm{a}} / \mathrm{P}_{\mathrm{b}}$, for two different species, $\mathrm{a}$ and $\mathrm{b}$, being transported across a biological membrane. Baker, et al, [5] have applied an adaptive multi-level finite element method (run on the NPACI Blue Horizon) to the solution of the Poisson-Boltzmann equation for a microtubule involved in cellular transport. An excellent review of the application of Poisson-Boltzmann calculations to biology is provided by Honig and Nicholls [6].

The usual starting point for these calculations is the solution of the Poisson-Boltzmann equation for which there is only one known analytic solution, that for an infinite flat plate or slab due to Gouy and Chapman [7] nearly a century ago. Most often, numerical solutions involving finite element methods such as those cited above, or finite difference 
methods (originating with Warwicker and Watson [8]) are employed, e.g., by Bruccoleri, et al [9], and recently by Luo, et al [10]. These latter authors have focused on the efficiency of the finite difference numerical method for solving the Poisson-Boltzmann equation, FDPB, and in eliminating the Coulomb cutoff employed in distance-dependent dielectric calculations. Gridding and mesh spacings become rather elaborate in these three dimensional numerical methods, which therefore struggle with sharp edges and corners. Bowen and Sharif [11] have applied an extensive adaptive mesh refinement method to solve the non-linear Poisson-Boltzmann equation in their study of pore entrance shapes in membrane design.

Alternatively, Green's function-based methods can be employed to reduce the dimensionality of the three dimensional problem to two-dimensional integrals over surfaces, but the methods are only formally applicable to the linearized form of the Poisson-Boltzmann equation, that is, to small potentials and charges. The method has been applied by Juffer, et al [12] to a molecule represented by atomic charges and by Wilson, et al [13] to couple the Schrödinger and Poisson-Boltzmann equations via the single- and double-layer surface charge distributions at a molecular-solvent interface. The linearized form is the starting point for Debye-Hückel theory [14] wherein the potential outside a sphere in an electrolyte is solved for.

In the Section II, we present our Method of Calculation involving direct summation over Debye-Hückel spheres (DHDS) on surfaces. The method is motivated by the Green's function approach, and as such, reduces the dimensionality of the solution to twodimensional summations. Also described in the Section II is the means to include charge redistribution in conducting surfaces held at constant potential. We validate the method in 
Section III by comparison to analytic solutions for a flat plate [7], a cylinder containing electrolyte [15] and a plane which contains a hole and is held at constant potential [16]. In Section IV, we apply the method to a mixed boundary condition problem, that of determining the potentials and ion concentrations in the vicinity of a nanomembrane. The nanomembrane is represented as a "spool-like" object whose cylindrical component is held at constant charge and whose "washer-like" components are held at constant potential. Section V contains Conclusions and a Discussion.

\section{Method of Calculation}

Electrostatic potentials in fluids are Coulomb potentials, screened by the presence of thermally diffuse counterions near charged surfaces and represented in diffuse equilibrium by the Poisson-Boltzmann equation [17],

$$
\nabla^{2} \phi=\frac{8 \pi n e v}{\varepsilon} \sinh (v e \phi / k T)
$$

where $\phi$ is the potential, $\mathrm{k}$ is Boltzmann's constant, T, the absolute temperature, $v$ the valence (here taken to be $v=1$ ), e the electronic charge, $n$ the density of ions far from the surface and $\varepsilon$ the dielectric constant of the fluid.

For potentials such that $v e \phi / k T<1$, the sinh can be expanded in a Taylor series; keeping the leading term in that series, the linearized form of the PB equation is obtained,

$\nabla^{2} \phi=\kappa^{2} \phi$

where $\kappa$ is the inverse Debye length, 
$\kappa^{2}=\frac{8 \pi n e^{2} v^{2}}{\varepsilon k T}$

\section{A. Direct summation (DHDS)}

We propose a method whereby solutions to the Poisson-Boltzmann equation for electrostatic potentials and fields can be readily obtained for sources in complex geometries and materials variations, and in any solution $\mathrm{pH}$ (varying natural surface charges) and ionic strength (including no salt, i.e., Laplace solutions). The method is furthermore capable of handling constant potential problems involving charge redistribution on conducting surfaces and finally, mixed boundary conditions. Sharp edges where electric fields are expected to be enhanced, and where three-dimensional finite element meshes must be exceptionally elaborate, are easily treated in this atomistic approach.

To this end, we take advantage of the atomic scale of the problem to build nanostructural surfaces atom-by-atom in arbitrary geometrical arrangements and then sum the potential due to each atom to find the total potential. We begin by assuming the potential, $\phi_{i}$, at a point $\mathrm{i}$ (either at an atomic surface or in the fluid) due to all other atoms, $\mathrm{j}$, has the form of the solution for a sphere in the linearized Poisson-Boltzmann approximation, i.e., the Debye-Hückel (DH) potential. We then directly sum (DS) the contributions from each atom (DHDS):

$\phi_{i}=\sum_{j} \frac{q_{j} \exp \left(-\kappa\left(r_{i j}-r_{0}\right)\right.}{\varepsilon r_{i j}\left(1+\kappa r_{0}\right)}$ 
where $q_{j}$ is the charge on atom $\mathrm{j} ; r_{i j}$ the distance between atom $\mathrm{i}$ and atom $\mathrm{j} ; \kappa$ is the inverse Debye length; $r_{0}$ the atomic radius (same for all atoms, $1.1 \AA$; representative of a typical nanomaterial, covalent $\mathrm{Si}$ ) and $\varepsilon$ is the dielectric constant $\left(78.5\right.$ for water at $25^{\circ}$ C).

Potentials determined in this way are then validated by comparison to known analytic solutions before being applied to more complex situations.

\section{B. Constant potential}

Charges on surfaces are naturally created by acid-base and surface complexation effects in aqueous electrolytic solutions (fixed charge boundary conditions). Alternatively, if the material comprising the nanostructure is a conductor and if the electrostatic potential at each atom in the surface is held at a constant value, surface charge will rearrange to accommodate this condition. A finite plane of atoms held at constant potential, for example, will build up surface charge near the edges of the plane; in the "washer-like" geometry we discuss below, a conductor will build up charge both in the rim of the hole in the washer and also on its outer peripheral atoms.

Consider a nanoengineered structure comprised of a conducting region of $\mathrm{N}_{\mathrm{I}}$ atoms interfacing directly to an insulating region of $\mathrm{N}_{\text {II }}$ atoms which is itself connected to a second conducting region of $\mathrm{N}_{\text {III }}$ atoms (a capacitor-like geometry; applicable to "spool" described below). The potential at an atom $\mathrm{i}$ in conducting region $\mathrm{I}$ is given by

$\phi_{\mathrm{i}}^{\mathrm{I}}=\mathrm{A}_{\mathrm{i}}+\sum_{\mathrm{I}} \mathrm{B}_{\mathrm{ij}} \mathrm{q}_{\mathrm{j}}^{\mathrm{I}}+\sum_{\mathrm{III}} \mathrm{C}_{\mathrm{ik}} \mathrm{q}_{\mathrm{k}}^{\mathrm{III}}$ 
where $A_{i}$ is the potential at $i$ due to all atoms in insulating region II (fixed charges); $q_{j}$ and $\mathrm{q}_{\mathrm{k}}$ are the charges on atoms in conducting regions I and III respectively; $\mathrm{B}_{\mathrm{ij}}$ and $\mathrm{C}_{\mathrm{ik}}$ are elements (see Eq. [4] above) of matrices corresponding to regions I and III, respectively.

Similarly, the potential at an atom $\mathrm{i}$ in conducting region III is given by

$$
\phi_{\mathrm{i}}^{\mathrm{III}}=\mathrm{D}_{\mathrm{i}}+\sum_{\mathrm{III}} \mathrm{F}_{\mathrm{ij}} \mathrm{q}_{\mathrm{j}}^{\mathrm{III}}+\sum_{\mathrm{I}} \mathrm{G}_{\mathrm{ik}} \mathrm{q}_{\mathrm{k}}^{\mathrm{I}}
$$

By defining,

$$
\boldsymbol{\Phi}=\left(\begin{array}{l}
\phi^{\mathrm{I}}-\mathbf{A} \\
\boldsymbol{\phi}^{\mathrm{III}}-\mathbf{D}
\end{array}\right) \quad \text { and } \quad \mathbf{Q}=\left(\begin{array}{l}
\mathbf{q}^{\mathrm{I}} \\
\mathbf{q}^{\mathrm{III}}
\end{array}\right)
$$

where $\boldsymbol{\phi}^{\mathrm{I}}, \mathbf{A}$ and $\mathbf{q}^{\mathrm{I}}$ are column vectors of length $\mathrm{N}_{\mathrm{I}}$, and $\boldsymbol{\phi}^{\mathrm{III}}, \mathbf{D}$ and $\mathbf{q}^{\mathrm{III}}$ are column vectors of length $\mathrm{N}_{\text {III. }}$.

The system of linear equations becomes,

$$
\boldsymbol{\Phi}=\left(\begin{array}{ll}
\mathbf{B} & \mathbf{C} \\
\mathbf{G} & \mathbf{F}
\end{array}\right) \mathbf{Q}
$$

where $\mathbf{B}, \mathbf{C}, \mathbf{G}$ and $\mathbf{F}$ are matrices whose elements are those in Eqs. [5] and [6]. Eq. [8] is solved by standard methods for the charges, $\mathbf{Q}$, given a fixed potential, $\boldsymbol{\Phi}$, on each atom in the conducting region.

Depending upon the specific geometry of the nanostructure and the Debye length of the electrolyte, these equations may simplify. That is, if the screening is such that atoms in 
one conductor do not contribute to the potential on the other, then Eqs. [5] and [6] each pertain to only one conductor.

\section{Validation of the Method}

\section{A. Comparison to Gouy-Chapman}

For small surface charges and potentials, the linearized form of the PB equation can be applied to determining the electrostatic potential. Assuming a Debye-Hückel form of potential from each atom comprising the surface, a comparison can be made between our direct summation method and the analytical solution, i.e., the Gouy-Chapman (GC) solution for an infinite plane. In order to determine if this functional form (Eq. [4]) of the potential is appropriate, we need to eliminate the effects of geometry, the finite vs infinite slabs, from the comparison. Of course, screening by counterions in the fluid reduces the extent of the potential contribution from each atom; the largest extent and the stringent comparison is therefore obtained in the no-salt limit, $\kappa=0$ [18].

In order to eliminate this geometrical effect from our comparisons, we employ a 30 atom x 30 atom plane to be used as a building block in constructing larger planes of atoms. With the z-axis through the middle of this central plane (red region in Fig. 1), we surround this plane (zone 0 ) with 8 similar planes to form zone 1 as shown in purple in Fig. 1. Zone 1 is then surrounded by 16 blocks to form zone 2 (blue); further zones are similarly added while maintaining the symmetry of the system about the z-axis. The size of the plane thus formed is $2 \mathrm{~N}+1 \times 2 \mathrm{~N}+1$ where $\mathrm{N}=$ number of zones included; the number of atoms in the plane is then $30(2 \mathrm{~N}+1) \times 30(2 \mathrm{~N}+1)$, corresponding to dimensions

$66(2 \mathrm{~N}+1) \AA \mathrm{x} 66(2 \mathrm{~N}+1) \AA$ if each atom is assigned a radius of $1.1 \AA(\sim 2.2 \mathrm{Bohr}, 0.11$ 
nm). A 100-zone plate therefore has the dimensions 13,266 $\AA$ x 13,266 $\AA$ (1.33 microns $x$ 1.33 microns) and contains over 36 million atoms. We estimate [18] that this should provide a $0.4 \%$ accuracy at $z_{a}=100 \mathrm{Bohr}$ in the no-salt $(\kappa=0)$ limit. For $\kappa>0.0$, screening due to the electrochemical double layer will significantly reduce the size of plane required to obtain a reasonable comparison with Gouy-Chapman.

Specifically, in Fig. 2a, we have plotted the electrostatic potential as a function of distance, $\mathrm{z}$, from a charged plane of density $\sigma=0.33 \mu \mathrm{C} / \mathrm{cm}^{2}$ for several values of $\mathrm{N}$, the number of zones of charged atoms in the plane. The calculations were performed in the no-salt $(\kappa=0)$ limit, that is, as solutions to the Laplace equation; the potential at $\mathrm{z}=0$ being fixed at the $\mathrm{N}=100$-zone value for all $\mathrm{N}$. The well-known analytic result for an infinite plane is that the electric field is constant $\left(=-\frac{2 \pi \sigma}{\varepsilon}\right)$ for this condition, that is, the potential decreases linearly. From Fig. $2 \mathrm{a}$ we find that for $\mathrm{N}=0$ (central plane alone), the potential falls off non-linearly with $\mathrm{z}$. For $\mathrm{N}>=5$, the potential for $\mathrm{z}<=225$ Bohr decreases linearly with a slope (shown in the inset) that rapidly converges to the value $-1.26 \times 10^{-4}$ volts $/$ Bohr $\left(=2.38 \times 10^{4}\right.$ volts $\left./ \mathrm{cm}\right)$. As shown in the inset in Fig. $2 \mathrm{a}$, the error involved in direct summation decreases asymptotically with the number of zones included in the calculation. With 5 zones, the slope is accurate to within $\sim 14 \%$, for 40 zones the accuracy is $\sim 2.3 \%$; at 100 zones, $<1 \%$. We therefore include 40 zones when making comparisons to the Gouy-Chapman $(\kappa>0)$ results, where screening is significant.

In Fig. $2 b$ we have plotted the ratio 
$R=\frac{\phi_{G C}-\phi_{D H D S}}{\phi_{G C}}$

where

$\phi_{G C}=\frac{2 k T}{v e} \ln \left\{\frac{1+\gamma \exp (-\kappa z)}{1-\gamma \exp (-\kappa z)}\right\}$

is the Gouy-Chapman potential, with

$\gamma=\tanh \left(\frac{v e \phi_{0}}{4 k T}\right)$

and $\phi_{0}$ is the surface electrostatic potential. $\phi_{D H D S}$ is the potential derived by direct summation for $\kappa=0.01 \mathrm{Bohr}^{-1}$ and charge density, $\sigma=0.33 \mu \mathrm{C} / \mathrm{cm}^{2}$. The value of the GC potential at $\mathrm{z}=0, \phi_{0}$, is fixed at the $\phi_{D H D S}$ value. It can be seen from Fig. $2 \mathrm{~b}$ that 5 zones results in a $0.25 \%$ error at 225 Bohr $(119 \AA, 11.9 \mathrm{~nm})$ whereas 10 or more zones result in agreement between our DHDS method and Gouy-Chapman of $<0.1 \%$.

In Fig. 3, the ratio R (Eq. [9]) is plotted as a function of charge density, fixing the number of zones, N, at 40 and the inverse Debye length at 0.01 Bohr $^{-1}$ (Fig. 3a) or 0.1 Bohr ${ }^{-1}$ (Fig. 3b). Note from Fig. 3a $\left(\kappa=0.01\right.$ Bohr $\left.^{-1}\right)$ that the DHDS method agrees with GouyChapman within $\sim 0.2 \%$ at a distance of 225 Bohr for charges densities, $\sigma$, of $33 \mu \mathrm{C} / \mathrm{cm}^{2}$ or less. When the charge density is $330 \mu \mathrm{C} / \mathrm{cm}^{2}$, the error is seen to increase with distance to a factor of $\sim 3$, but is a virtual constant beyond the Debye length of 100 Bohr. At $\sigma=$ $3300 \mu \mathrm{C} / \mathrm{cm}^{2}$, the error is a factor of $\sim 45$. This is caused by a fundamental limitation of the method: because it is based on the solution to a Debye-Hückel sphere, one expects the 
result to diverge from the correct Gouy Chapman potential for large charges as the Taylor series expansion of the sinh term above (Eq. [1]) suggests.

From Fig. 3a, the DHDS method agrees with Gouy Chapman to $<=0.1 \%$ at an inverse Debye length of $\kappa=0.01 \mathrm{Bohr}^{-1}$ for charge densities up to $\sigma=33 \mu \mathrm{C} / \mathrm{cm}^{2}$. In Fig. 3b, we have plotted similar comparisons for $\kappa=0.1 \mathrm{Bohr}^{-1}$. The screening due to counterions is now great enough that even for charge densities as large as $\sigma=330 \mu \mathrm{C} / \mathrm{cm}^{2}$ the direct summation (DHDS) method agrees within $\sim 6 \%$ with Gouy-Chapman.

We conclude that the potential derived by the direct summation method agrees quite well with that of Gouy-Chapman (with geometrical considerations accounted for) depending on the Debye length and atomic charges employed. We conservatively restrict ourselves to charge densities, $\sigma$, less than $33 \mu \mathrm{C} / \mathrm{cm}^{2}$ on each atom in the following calculations.

\section{B. Validation by comparison to analytic solution for cylinder}

We construct rings of radius $\mathrm{R}$ from atoms of radius $\mathrm{r}_{0}$, each atom having charge $\mathrm{q}$. The rings are stacked touching, not overlapping, to form a cylinder of length $\mathrm{L}$. We then applied our DHDS method to the calculation of the potential and electric field within the cylinder, recognizing that there will be edge effects (as will exist in the real world). We then compare these calculations to the known analytic results.

The analytic solution for the electrostatic potential in an infinite cylinder containing electrolyte in the linearized Poisson-Boltzmann equation approximation has been shown to be $[15]$ 
$\phi_{c y l}=\phi_{c y l}^{0} \frac{\mathrm{I}_{0}(\kappa r)}{\mathrm{I}_{0}(\kappa a)}$

where $I_{0}$ is the zero-order modified Bessel function of the first kind and $r$ is the radial distance from the axis of the cylinder whose radius is a.

In Fig. 4, we show the comparison between the methods for a $50 \AA$ (5 nm) and $100 \AA$ (10 nm) diameter cylinder, $200 \AA$ in length, whose inner walls are held at a constant potential (in the analytic solution) consistent with a charge density of $\sigma=2.10 \mu \mathrm{C} / \mathrm{cm}^{2}$. The potential is calculated along a line through the center of the cylinder. The excellent agreement between the DHDS method and the Bessel function solution displayed in Fig. 4 is encouraging.

\section{Validation by comparison to analytic solution for hole in plane}

Here we treat a nanostructural component, a "washer-like" geometry consisting of a ring of atoms forming a conductor of outer radius $\mathrm{R}$ containing a centrally located hole of radius a. The washer is held at a constant potential of $100 \mathrm{mV}$. We use the linear algebraic method described in Section I above to calculate the charge on each atom in a washer of inner radius $\mathrm{a}=25 \AA$ and outer radius $\mathrm{R}=100 \AA$. These potentials are compared to the analytical solution for an infinite plane containing a hole.

Jackson [16] writes the potential at a point at distance $\mathrm{z}$ from an infinite conducting plane containing a hole of radius a as

$$
\begin{aligned}
& \Phi=E_{0} z+\Phi^{(1)} \ldots \ldots . . z>0 \\
& \Phi=E_{1} z+\Phi^{(1)} \ldots \ldots . . z<0
\end{aligned}
$$


where $E_{0}$ and $E_{1}$ are specified asymptotic fields and $\Phi^{(1)}$ is the potential due to the rearrangement of charge near the hole, expressible as

$\Phi^{(1)}(\rho, z)=\int_{0}^{\infty} d k A(k) \exp (-k|z|) J_{0}(k \rho)$

where $J_{0}$ is a Bessel function of order zero. After some manipulation, the potential is found to be

$\Phi^{(1)}(\rho, z)=\frac{\left(E_{0}-E_{1}\right) a}{\pi}\left\{\left[\frac{R-\lambda}{2}\right]^{\frac{1}{2}}-\frac{|z|}{a} \tan ^{-1}\left(\frac{2}{R+\lambda}\right)^{\frac{1}{2}}\right\}$

where

$\lambda=\frac{z^{2}+\rho^{2}-a^{2}}{a^{2}} \quad$ and $\quad R=\left(\lambda^{2}+\frac{4 z^{2}}{a^{2}}\right)^{\frac{1}{2}}$

The field, $E_{0}$, to be specified in Eq. [15] above is asymptotically $\frac{2 \pi \sigma}{\varepsilon}(\kappa=0$ here); the surface charge density, $\sigma$, is then $2.17 \times 10^{-4} \mathrm{e} / \mathrm{Bohr}^{2}\left(0.79 \mu \mathrm{C} / \mathrm{cm}^{2}\right)$ on atoms in the midpoint region between the inner and outer rims of the washer (from the solution to Eq. [8]). With $\varepsilon=78.5$ (water), $E_{0} \sim 1.105 \times 10^{-5} \mathrm{e} / \mathrm{Bohr}^{2}\left(5.7 \times 10^{4}\right.$ volts $\left./ \mathrm{cm}\right)$. Here $E_{1}=-E_{0}$.

In Fig. 5, we compare the potential along the z-axis normal to the plane of the washer calculated by our DHDS method with the analytic solution using Eqs. [13] and [15]. Our numerical DHDS method is seen to agree quite well with the analytic result up to $\mathrm{z} \sim 200$ Bohr $(\sim 10.6 \mathrm{~nm})$ where the finite size of our washer (outer diameter $\sim 400$ Bohr) causes 
the solution to differ from the result for the infinite plane. At this distance, the potential bends over as expected; at infinity the washer acts like a point charge. Note the analytic solution at large distances is a straight line, the expected potential for an infinite charged plane, the effect of the hole diminishing with $\mathrm{z}$. The inset shows a contour plot of the DHDS potential in the $\mathrm{x}-\mathrm{z}$ plane perpendicular to the washer.

In Fig. 6, we plot the potentials as a function of $x$ at several values of $z$ (heights above the plane) for $\kappa=0.0$. The lower value of $z$ chosen is at the radius of the atoms in the DHDS washer; the agreement with the analytic solution is, again, quite encouraging. For $\mathrm{z}=25.0$ Bohr, the potentials agree for $\mathrm{x}$ values appropriate to the hole, $|x| \leq 50 \mathrm{Bohr}$, but the charge pileup on the outer rim of the washer creates a curving upward of the DHDS potential for values of $\mathrm{x}$ outside the region of the hole, as expected. At $\mathrm{z}=175 \mathrm{Bohr}$, the potentials compare similarly: somewhat poorer agreement in the hole region than for 25 Bohr, but again a turning up of the DHDS potential due to the outer rim of charge. The inset shows the potential in a plane parallel to the washer at this same $\mathrm{z}=2.078 \mathrm{Bohr}$ height.

In the presence of an electrolyte of inverse Debye length, $\kappa$, an infinite plane held at constant potential will adjust its surface charge density, $\sigma$, so as to maintain a constant ratio of $\sigma$ to $\kappa$. That is [17],

$$
\phi=\frac{4 \pi \sigma}{\varepsilon \kappa}
$$

An interesting result is obtained if a conducting circular disk (washer without the hole) is considered to be immersed in an electrolyte and the potential employed in solving Eq. [8] 
assumed to be the screened Coulomb, Debye-Hückel potential of Eq. [2] with $\varepsilon=100$, appropriate to a metal. For $0.005<\kappa<0.1$, the charge density on the inner region, $|x| \leq 50$ Bohr of atoms (far away from the outer rim charge pileup) divided by the inverse Debye length is found to be a constant within $\sim 3 \%$, in essential agreement with Eq. [17]. We are pursuing this interesting effect further [19].

We next applied our method to the washer above, held at constant $100 \mathrm{mV}$ potential, for $\kappa=0.0,0.01$ and $0.1 \mathrm{Bohr}^{-1}$ for solutions containing salt and find charge to be built up at the inner and outer rims for all Debye lengths. Charge density, $\sigma$, at the mid-point of the washer increases $\left(0.84 \mu \mathrm{C} / \mathrm{cm}^{2}, 2.1 \mu \mathrm{C} / \mathrm{cm}^{2}, 20 \mu \mathrm{C} / \mathrm{cm}^{2}\right)$ with increasing inverse Debye length $\left(0.0,0.01,0.1 \mathrm{Bohr}^{-1}\right.$, respectively) in order to maintain constant potential, consistent with the result above for the circular disk. Charge buildup at the inner rim atoms $\left(2.1 \mu \mathrm{C} / \mathrm{cm}^{2}, 6.51 \mu \mathrm{C} / \mathrm{cm}^{2}, 42.2 \mu \mathrm{C} / \mathrm{cm}^{2}\right)$ and outer rim atoms $\left(6.3 \mu \mathrm{C} / \mathrm{cm}^{2}\right.$, 13.4 $\mu \mathrm{C} / \mathrm{cm}^{2}, 45.4 \mu \mathrm{C} / \mathrm{cm}^{2}$ ) also increases with Debye length, 0.0, 0.01, 0.1 $\mathrm{Bohr}^{-1}$, respectively, where Eq. [17] is not applicable.

In Fig. 7 we have plotted the magnitude of the electric field for $\kappa=0.01 \mathrm{Bohr}^{-1}$ in the $\mathrm{z}=4$ Bohr plane parallel to the plane of the washer (looking down). In Fig. 7a, the atoms in the washer are held at a fixed charge density of $2.1 \mu \mathrm{C} / \mathrm{cm}^{2}$, whereas in Fig. $7 \mathrm{~b}$, each atom in the washer is held at constant potential, $100 \mathrm{mV}$, and the charges allowed to rearrange, that is, the washer is a conductor. The charge density near the middle of the washer is 2.1 $\mu \mathrm{C} / \mathrm{cm}^{2}$ under the constant potential conditions, providing a meaningful comparison to Fig.7a. Note the dramatic increase in field strength (red "ring" region) caused by the 
charge buildup at the inner rim of the hole in Fig. 7b. The insets in Fig. 7 provide the magnitude of the electric field as the third dimension and provide further graphical evidence of this insulator vs conductor effect.

It is also to be noted in Fig. 7 that, at this ionic strength, the electrochemical double layers from each side of the inner rim are overlapping; the potential at the center is not screened out.

\section{Application to Nanomembrane}

Having validated our computational DHDS method for cylinder and washer geometries, we now assemble two washers connected by a cylinder into a spool, a model of a nanopore whose top and bottom surface may be constructed of different materials from the cylinder. For example, with the cylinder made of an insulator such as silica or alumina, the top and bottom surfaces may be either insulating (perhaps same material as the cylinder) or conducting (gold or silver plated). Of course, it is recognized that in this skeletal model of a membrane pore, polarizable membrane material actually exists in the intervening region between the cylinder walls and washer. Charges on the surface will induce polarization charge at the interface [20-22] which, in turn, gives rise to a perturbing potential in the region of interest. These effects are outside the scope of this paper.

In Fig. 8, we compare the potentials and positive and negative ion distributions for spools having constant surface charge with those whose conducting (washer) regions are held at constant potential. For comparison purposes, we keep the same scale for each of these quantities, i.e., potential, positive ions and negative ions, as shown at the bottom of the 
figure. In Fig. 8a-c, we have plotted the potentials and concentrations of positive and negative ions in the vicinity of an insulating spool, each atom of which (washer and cylinder) is held at constant charge density, $1.05 \mu \mathrm{C} / \mathrm{cm}^{2}$. Note the increase in potential near the top of the cylinder but below the plane of the washer (red region in Fig. 8a). This is explained as follows: The potential near the cylinder wall from the middle plane of the cylinder upward along the z-axis due to the charges on the cylinder alone is a constant (yellow region in Fig. 8a) until the number of atoms contributing to that potential decreases near the top of the cylinder. Beyond this point, the potential decreases rapidly. The potential along the same line due to charges on the washer alone rises monotonically, peaks at the plane of the washer and then falls with the same slope. In the red region of Fig. $8 \mathrm{a}$, it is seen that the peak of the total potential is shifted to below the plane of the washer by the contribution from charge on the cylinder.

It is also to be noted (in Fig 8b) that positive ions are seen to be rejected by this spool of fixed charge, an expected result which makes Fig. 8b rather boring looking on this scale. Positive ions in concentrations of less than $10^{18}$ actually exist but are not observed on this scale. Negative ions (Fig. 8c) are concentrated in regions where the potential is a maximum (Fig. 8a).

In Fig. 8d-f, we show a similar set of results for a spool whose washer elements are conductive and held at $+/-100 \mathrm{mV}$, while the cylinder connecting them is treated as an uncharged insulator. The potential, Fig. 8d, has its maximum in the plane of the washer unlike the fixed charge case (Fig.8a); here the potential is due only to the buildup of charge on the rim of the washer, there can be no shifting of the maximum downward as 
we have seen in Fig. 8a because there is no charge on the cylinder. In Fig. 8e, the positive ion concentration is seen to be rejected by the positive potential on the top (we present further details on an expanded scale in Fig. 9 below) while it is enhanced by the negative potential applied on the bottom. In Fig. 8f, the opposite effect is seen: The negative ion concentration is seen to be rejected by the negative potential on the bottom while it is enhanced by the positive potential applied on the top.

In Fig. 8g-i, the washer elements are held at $+/-100 \mathrm{mV}$ and the charge density on the atoms comprising the cylinder is held constant at $1.05 \mu \mathrm{C} / \mathrm{cm}^{2}$. This configuration results in an asymmetry in the potential and ion concentrations. The maximum in the potential at the top is shifted below the plane of the washer (Fig. $8 \mathrm{~g}$ ) because of the charge on the cylinder coming into play, much like in Fig. 8a. In fact, the positive cylinder charge results in a lowering of the charge on the inner rim atoms of the top washer (from Eq. [8]). Also shown in Fig. 8g at the bottom, the potential maximum is now in the plane of the bottom washer. Here the positive cylinder charge results in an increase in the charge on the inner rim atoms of the washer, being held at a constant negative potential.

In Fig. 8h, the positive ion concentration is seen to be rejected by the positive potential at the top while it is enhanced by the negative potential applied on the bottom: the positive ion concentration is rejected to a greater extent than is observed in Fig. 8e. In Fig. 8i, the negative ion concentration at the top of the cylinder is seen to be more enhanced than in Fig. $8 \mathrm{f}$ due to the charges on the cylinder atoms. The negative ions are rejected by the negative potential on the bottom but to a lesser extent than in Fig. $8 \mathrm{f}$ because the negative applied potential there is mitigated by the positive charges on the cylinder. 
Finally, in Fig. 9 we compare the positive and negative ion distributions in a limited region above the washer for a constant charge density $\sigma=2.1 \mu \mathrm{C} / \mathrm{cm}^{2}$ and a constant positive potential of $100 \mathrm{mV}$. Arrows indicate the position of the hole. Note the increased rejection of positive ions in the constant potential case created by the buildup of charge in the inner rim of the washer. Note also the larger attraction of negative ions in the constant potential case.

\section{Conclusions and discussion}

We have developed a method of calculation of the electrostatic potentials and fields in the vicinity of geometrically complex engineered nanostructures comprised of varying materials by direct summation of charged Debye-Hückel spheres (DHDS). By including charge redistribution on the surface of conducting materials held at constant potential, the method is applicable to mixed boundary conditions. We validated the method by direct comparison to analytical solutions for an infinite plane (Gouy-Chapman), an infinite cylinder (Bessel functions) and an infinite plane which contains a hole and which is held at constant potential. Excellent agreement between the potentials obtained by our numerical method and the closed form solutions is found over a wide range.

It is interesting to note that the DHDS method is applicable to those problems involving surfaces having charges which are separated by distances in excess of the Debye length. Such localized charge configurations are difficult to address by finite element or finite difference methods which then must finely mesh the local region surrounding each charge in order to find the potential at some arbitrary point in space. The DHDS method quite naturally calculates this potential as a sum over the charges. 
The DHDS method is applied to the calculation of the electric field enhancement in the vicinity of a nanomembrane whose cylindrical component is held at constant charge and whose "washer-like" ends are held at constant potential. The electric field is found to be enhanced by the charge buildup in the rim of the hole of the nanomembrane, the redistribution of which results from the potential being held constant in the conducting region. Ion concentrations are also calculated; positive ion rejection is found to be enhanced by this charge buildup in the region of the rim when a constant positive potential is applied.

Our central result: Constructing the engineered membrane top and bottom surfaces from a conducting (metal) rather than insulting (ceramic) material can give rise to enhanced electric fields at the inlet to the nanopore. Conduction in the top and bottom surfaces, by allowing for the redistribution of charge to the inner rims of the holes, results in altered ion distributions in the inlet and outlet regions of a nanomembrane. 


\section{References}

1. U.S. EPA (2002) List of contaminants and their maximum contaminant levels, Rpt. No. EPA 816-F-02-013 (2002).

2. Wilbert, M.C., Leitz, F., Abart, El, Linton, K., Water Treatment Technology Program Report No. 29, Rpt \# R-98-05, U.S. Dept. of Interior (1998).

3. Tieleman, D.P., Borisenko, V, Sansom, M.S.P. Woolley, G.A., Biophys. J. 84 (2003) 1464.

4. Miedema, H., Biophys. J. 82 (2002) 156.

5. Baker, N.A., Sept, D., Holst, M.J., McCammon, J.A., IBM J. Research Develop. 45 (2001) 427.

6. Honig, B., Nicholls, A., Science 268 (1995) 1144.

7. Gouy, G., J. Physique 9 (1910) 457; also, independently, Chapman, D.L., Phil. Mag. 25 (1913) 475.

8. Warwicker, J., Watson, H.C., J. Mol. Biol. 157 (1982) 671.

9. Bruccoleri, R.E., Novotny, J., Davis, M.E., Sharp, K., J. Comput. Chem. 18 (1997) 268.

10. Luo, R., David, L., Gilson, M.K., J. Comput. Chem. 23 (2002) 1244.

11. Bowen, W.R., Sharif, A.O., Colloids and Surfaces A, 201 (2002) 207. 
12. Juffer, A.H., Botta, E.F.F., van Keulen, B.A.M., van der Ploeg, A., Berendsen, H.C., J. Comput. Phys. 97 (1991) 144.

13. Wilson, W.D., Schaldach, C.M., Bourcier, W.L., Chem. Phys. Letters, 267 (1997) 431.

14. Debye, P., Hückel, E., Physik Z. 24 (1923) 185.

15. Rice, C.L, Whitehead, R.J., Phys. Chem. 69 (1965) 4017. See also, Rice, R.E., Horne, F.H., J. Chem. Phys. 75 (1981) 5582; Rice, R.E., J. Chem. Phys. 82 (1985) 4337.

16. Jackson, J.D., Classical Electrodynamics, 3rd ed., John Wiley and Sons, New York, 1999.

17. Verwey, E.J.W., Overbeek, J.TH.G., Theory of the Stability of Lyophobic Colloids, Elsevier, New York, 1948.

18. In the $\kappa=0$ limit, it is simple to estimate how large the flat plate must be for the direct summation to be compared to the infinite solution: the electric field, $E_{z}$, at a point, $z_{a}$, along the z-axis normal to a charged plate (surface charge density, $\sigma$ ) of radius $\rho$ is simply,

$$
E_{z}=\frac{2 \pi \sigma}{\varepsilon}\left(1-\frac{z_{a}}{\left(z_{a}^{2}-\rho^{2}\right)^{\frac{1}{2}}}\right)
$$


When $\rho \rightarrow \infty$, the familiar expression $E_{z}=-\frac{2 \pi \sigma}{\varepsilon}$ is obtained. At $z_{a}=10(100) \mathrm{Bohr}$, a $10^{4} \AA$ radius disk will create a field differing from the infinite disk value by less than $0.1 \%(0.5 \%)$

19. We are applying a Green's function-based method [13] to study metal clusters in this investigation.

20. Wilson, W.D., Schaldach, C.M., J. Colloid Interface Sci. 208 (1998) 546.

21. Wilson, W.D., Bisson, C.L., Schaldach, C.M., J. Colloid Interface Sci. 187 (1997) 201.

22. Wilson, W.D., Schaldach, C.M., Proceedings of the $191^{\text {st }}$ Meeting of the Electrochemical Society, Montreal, Quebec, Canada, May 4-9,1997. 


\section{Figure Legends}

Figure 1: Zoning scheme employed in making comparison with Gouy-Chapman. A building block (red) is used to create zone 1 by surrounding the central block with first neighbors (purple); zone 2 is created by constructing second neighbors (blue), etc. The electrostatic potential as a function of $\mathrm{z}$ is calculated by direct summation over DebyeHückel (DH) spheres and also using the method of Gouy-Chapman (see Figs. 2-3).

Figure 2: Comparison of potential obtained by direct summation method (DH) with analytic results for an infinite charged plane of density $\sigma=0.33 \mu \mathrm{C} / \mathrm{cm}^{2}$. Fig. 2a) With no screening due to counterions (Laplace equation), it is to be noted that at 100 zones the error is $<0.5 \%$; for 40 zones, the error is $\sim 3 \%$. Fig. $2 b)$ The ratio, $R=\left(\phi_{\mathrm{GC}}-\phi_{\mathrm{DH}}\right) / \phi_{\mathrm{GC}}$ is plotted as a function of distance along the z-axis for different numbers of zones (see Fig. 1). At a Debye length of 100 Bohr, agreement between our DHDS method and GouyChapman is $<=0.1 \%$ even when only 10 zones are employed.

Figure 3: Ratio R (Eq. [9]) is plotted as a function of charge density on individual spheres, fixing the number of zones, $\mathrm{N}$, at 40 and the inverse Debye length at $0.01 \mathrm{Bohr}^{-1}$ (Fig. 3a) or 0.1 Bohr $^{-1}$ (Fig. 3b). The method agrees with Gouy-Chapman within $0.1 \%$ for charge densities $\sigma=33.0 \mu \mathrm{C} / \mathrm{cm}^{2}$ for both Debye lengths shown.

Figure 4: Comparison of present method of calculation (DHDS) for a cylinder of length $200 \AA$ with analytic solution for an infinite cylinder whose inner walls are held at a constant potential consistent with a charge density $\sigma=2.1 \mu \mathrm{C} / \mathrm{cm}^{2}$. The potential is 
calculated along a line through the center of the cylinder. Note the excellent agreement between the DHDS method and the Bessel function solution.

Figure 5: Electrostatic potential along the z-axis normal to the plane of a washer calculated by the present DHDS method and also by the analytic method (see Eqs. [13] and [15]) for $\kappa=0.0 \mathrm{Bohr}^{-1}$. Note the excellent agreement for $\mathrm{z}<\sim 150 \mathrm{Bohr}$ beyond which the finite nature of the washer used in the DHDS calculations causes the DHDS solution to diverge from the result for an infinite plane. The inset shows a contour map of the DHDS potential in the $\mathrm{x}-\mathrm{z}$ plane perpendicular to the washer.

Figure 6: Electrostatic potential as a function of $\mathrm{x}$ at several values of $\mathrm{z}$ (heights above the plane of the washer) for $\mathrm{\kappa}=0.0 \mathrm{Bohr}^{-1}$ calculated by the present DHDS method and also by the analytic method (see Eqs. [13] and [15]). Note the excellent agreement along the washer surface $(\mathrm{z}=2.078 \mathrm{Bohr})$. For $\mathrm{z}=25.0 \mathrm{Bohr}$, the potentials agree for $\mathrm{x}$ values appropriate to the hole, but the charge pileup on the outer rim of the washer creates a curving upward of the DHDS potential for values of $\mathrm{x}$ outside of the region of the hole, as expected. At $\mathrm{z}=175 \mathrm{Bohr}$, the potentials compare similarly somewhat poorer agreement in the hole region than for $25 \mathrm{Bohr}$, but again a turning up the DHDS potential due to the outer rim of charge. The inset shows the potential in a plane parallel to the washer at the $\mathrm{z}=2.078$ Bohr height.

Figure 7: Electrostatic potential and field in a plane parallel to that of the washer and at height of 4.0 Bohr $(0.2 \mathrm{~nm})$ for an inverse Debye length of $\kappa=0.01 \mathrm{Bohr}^{-1}$. In Fig. 7a, the charge density is fixed at $\sigma=2.1 \mu \mathrm{C} / \mathrm{cm}^{2}$, while, in Fig. $7 \mathrm{~b}$, the potential on each atom is fixed at $100 \mathrm{mV}$. Note the dramatic increase in field strength (red "ring" region) caused 
by the charge buildup at the inner rim of the hole in Fig. $7 \mathrm{~b}$. The insets provide the magnitude of the electric field as the third dimension and provide further graphical evidence of this insulator vs conductor effect.

Figure 8: Electrostatic potential and ion distributions in the vicinity of a spool. Fig. 8a-c pertains to an insulating spool, each atom of which is held at constant charge density, $\sigma=$ $1.05 \mu \mathrm{C} / \mathrm{cm}^{2}$. In Fig. 8d-f, the washer elements of the spool are conductive and held at $+/$ $100 \mathrm{mV}$, while the cylinder connecting them is treated as an insulator with zero atomic charges. In Fig. 8g-i, the washer elements of the spool are conductive and held at $+/-100$ $\mathrm{mV}$, while the cylinder connecting them is treated as an insulator with charge density $\sigma=$ $1.05 \mu \mathrm{C} / \mathrm{cm}^{2}$. Note that the charge redistribution in the conductive spool dramatically enhances the potential, and hence the ion distributions, in the vicinity of the top and bottom of the cylinders (rims of the washer elements) of the spool. Scales given at the bottom are held constant for each quantity for comparison purposes. See Fig. 9 for further details (on an expanded scale for top region) of Fig. 8e.

Figure 9: Positive and negative ion distributions in a limited region above the washer for constant charge and constant positive potential $(100 \mathrm{mV})$. Arrows indicate the position of the hole. Note the increased rejection of positive ions in the constant potential case created by the buildup of charge in the inner rim of the washer. Note also the larger attraction of negative ions in the constant potential case. 





$\mathbf{K}=0.0$

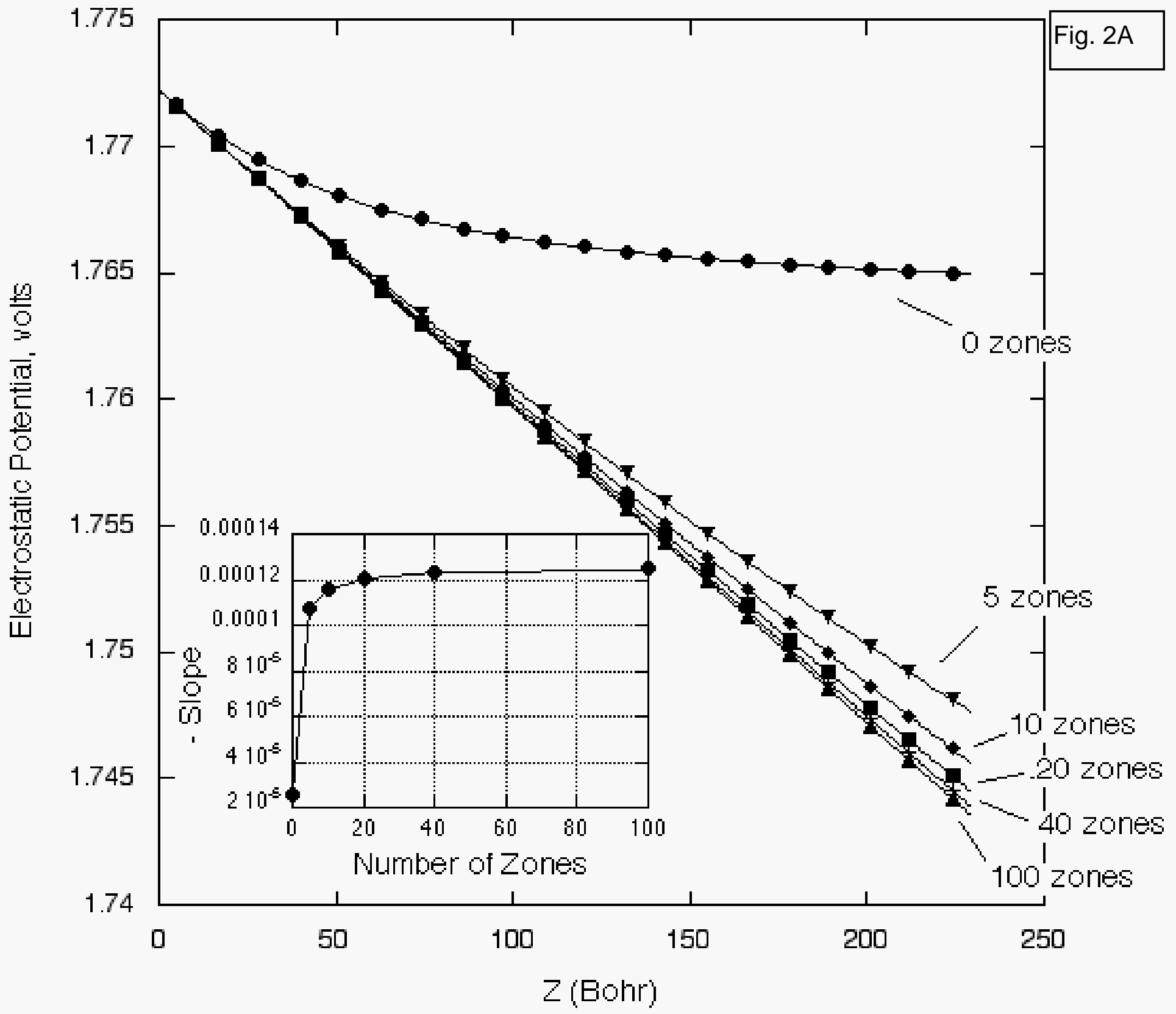




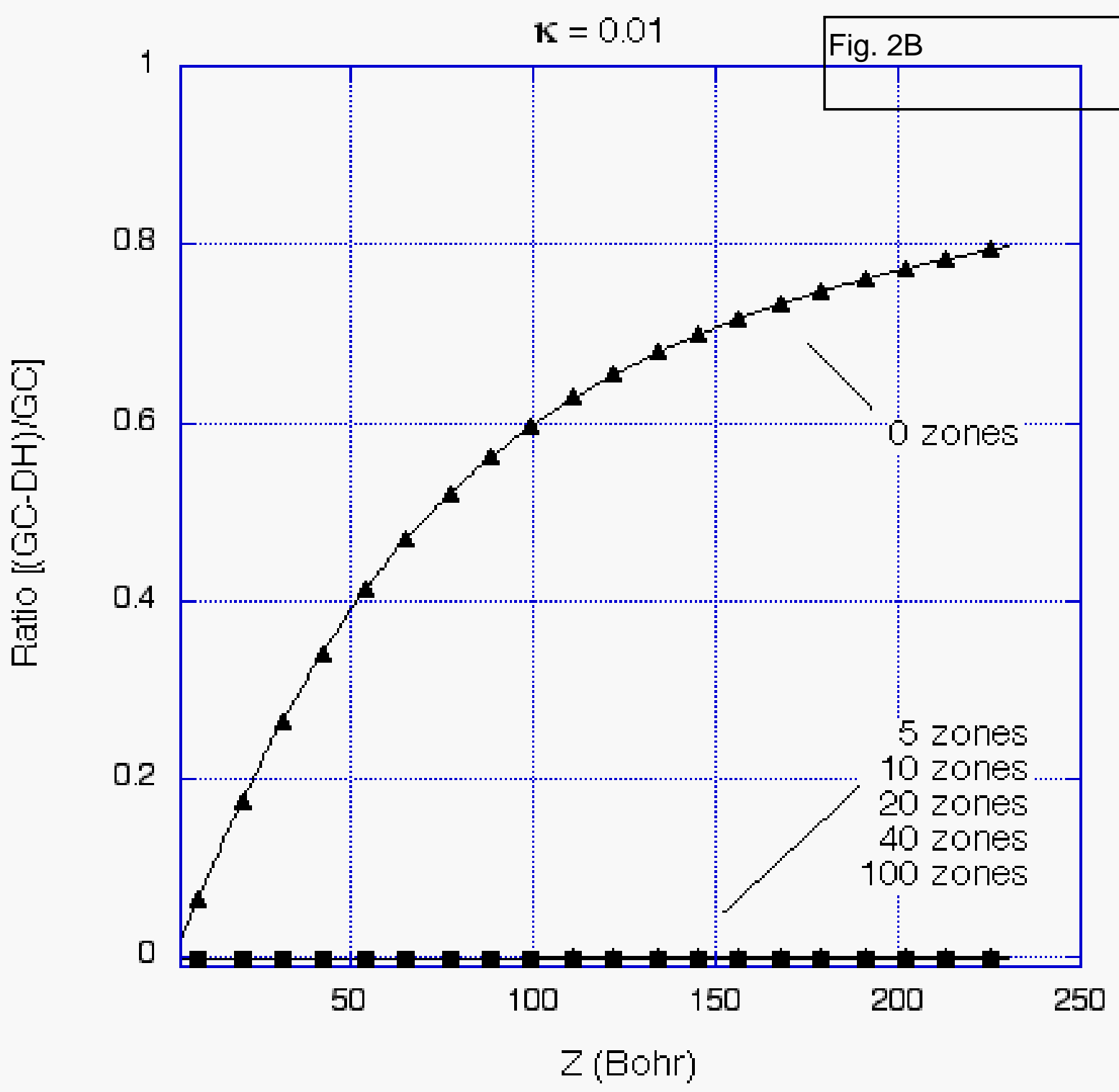




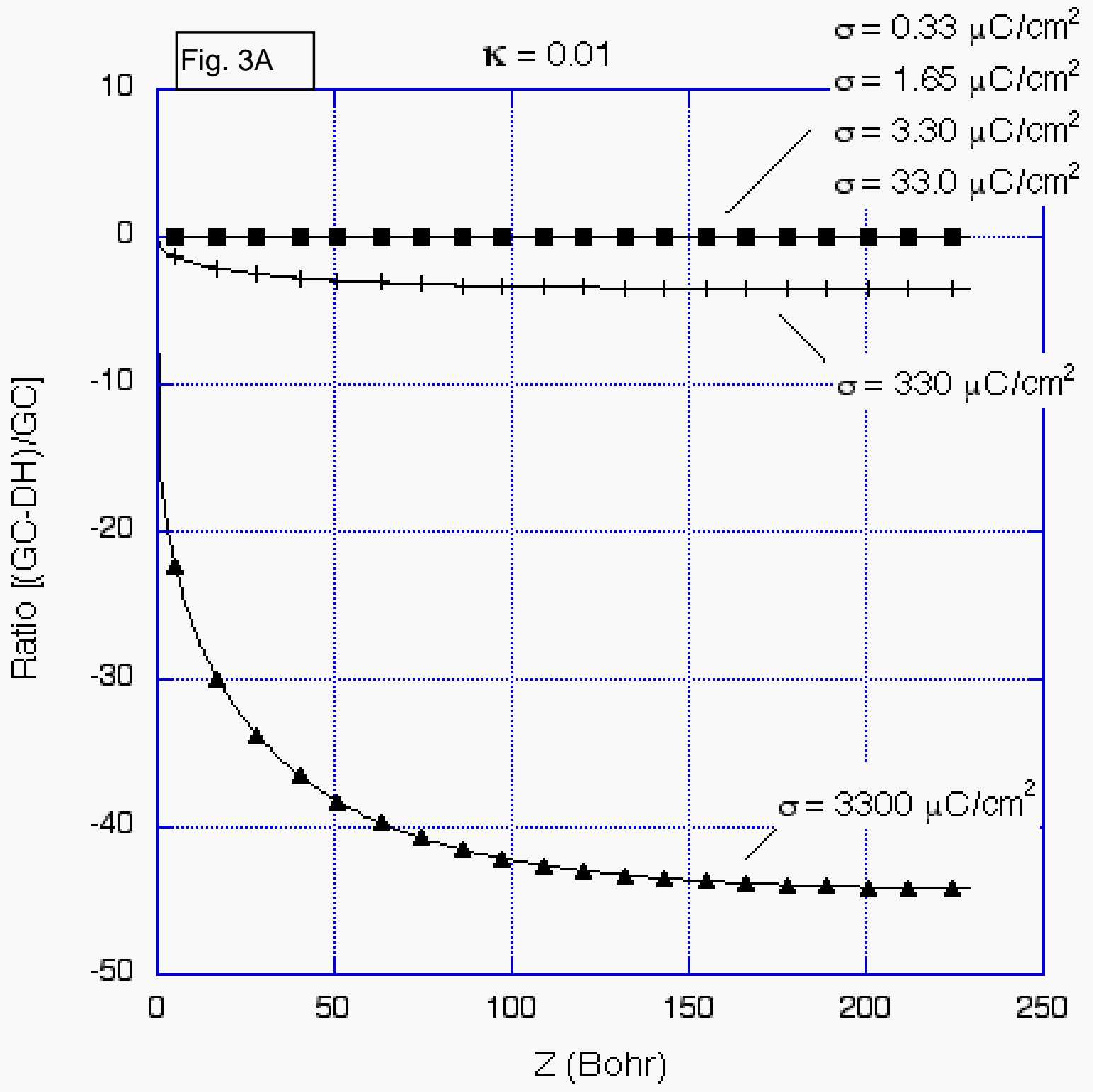




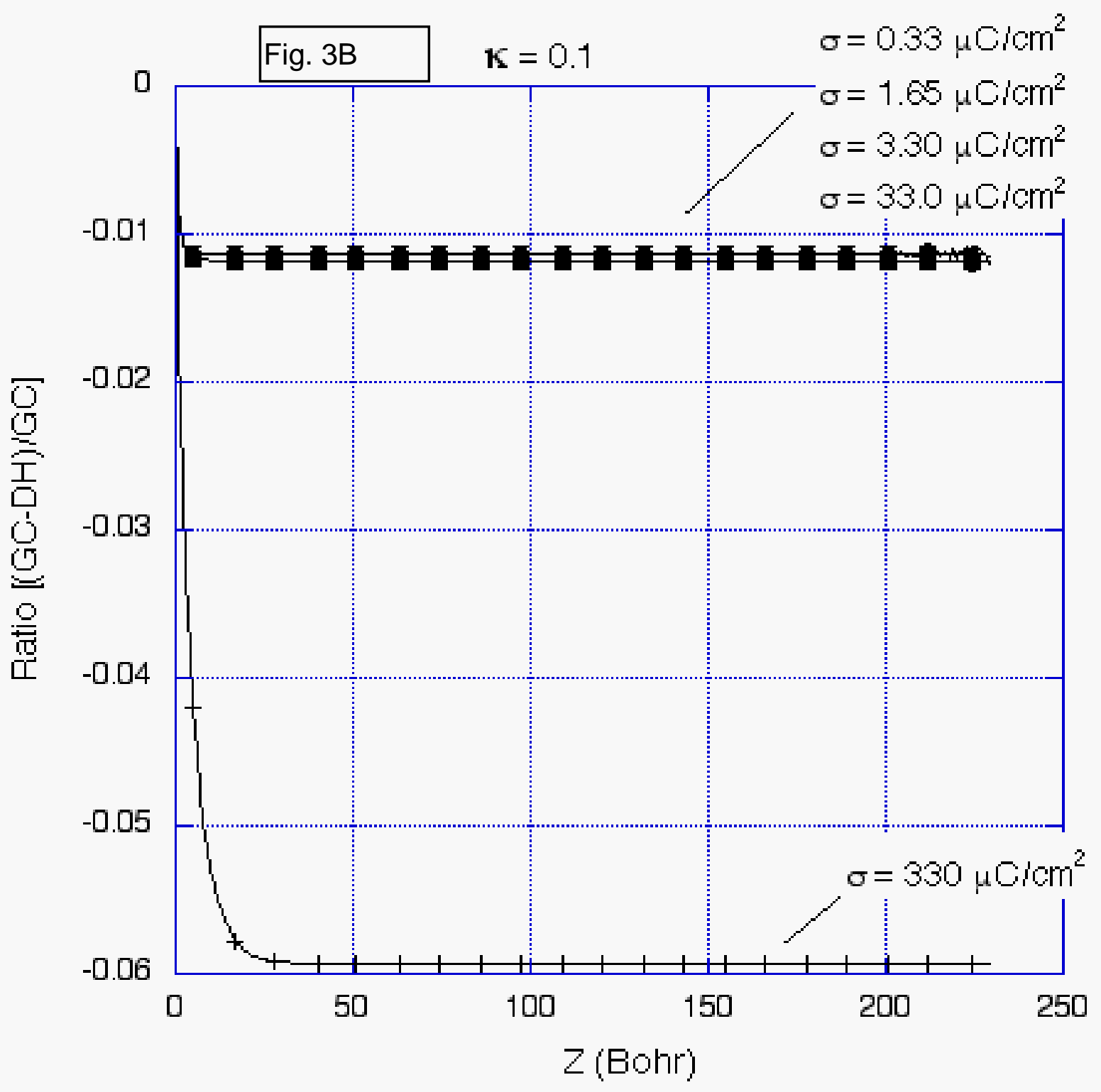



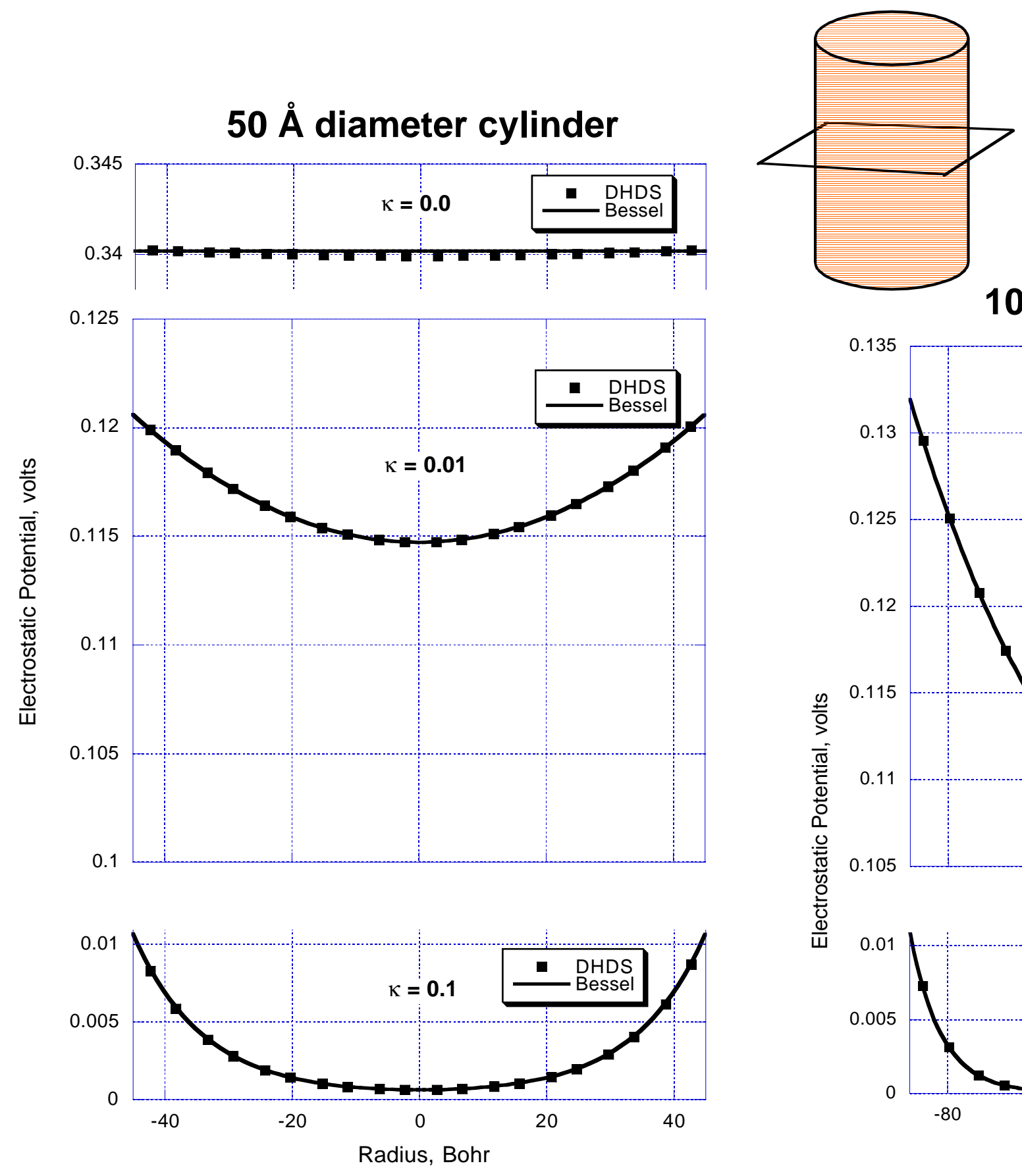

100 Å diameter cylinder

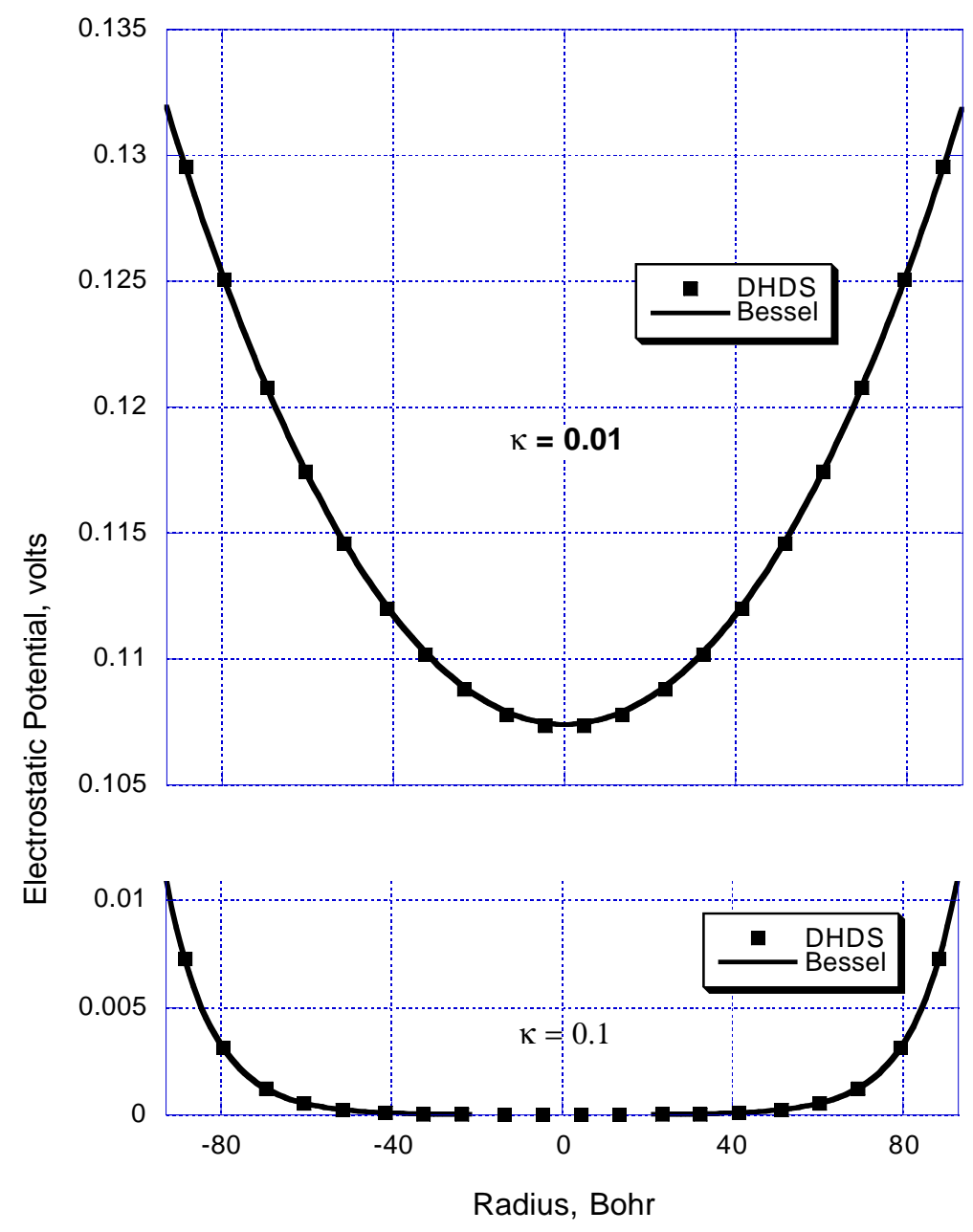




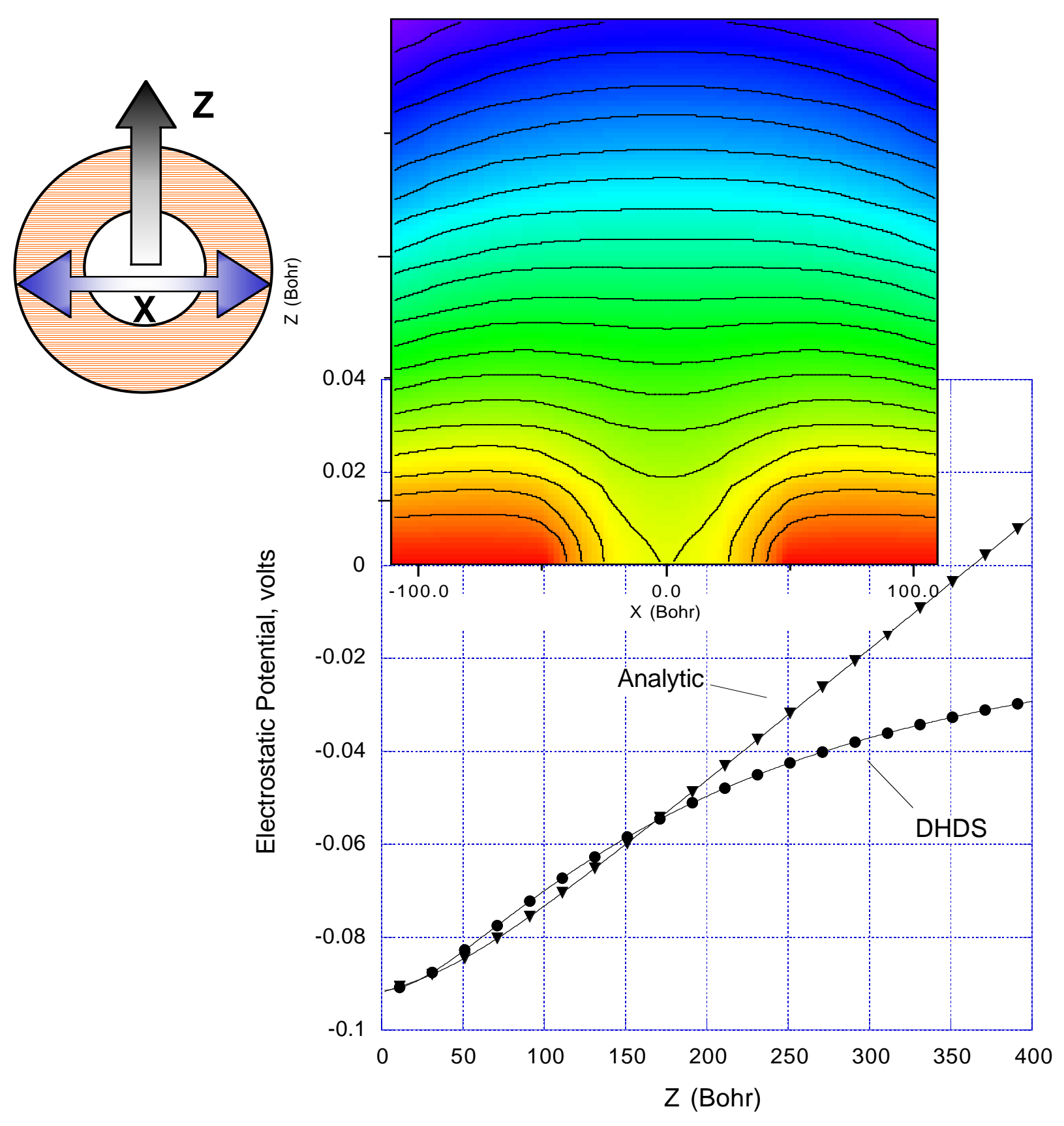

Fig. 5 


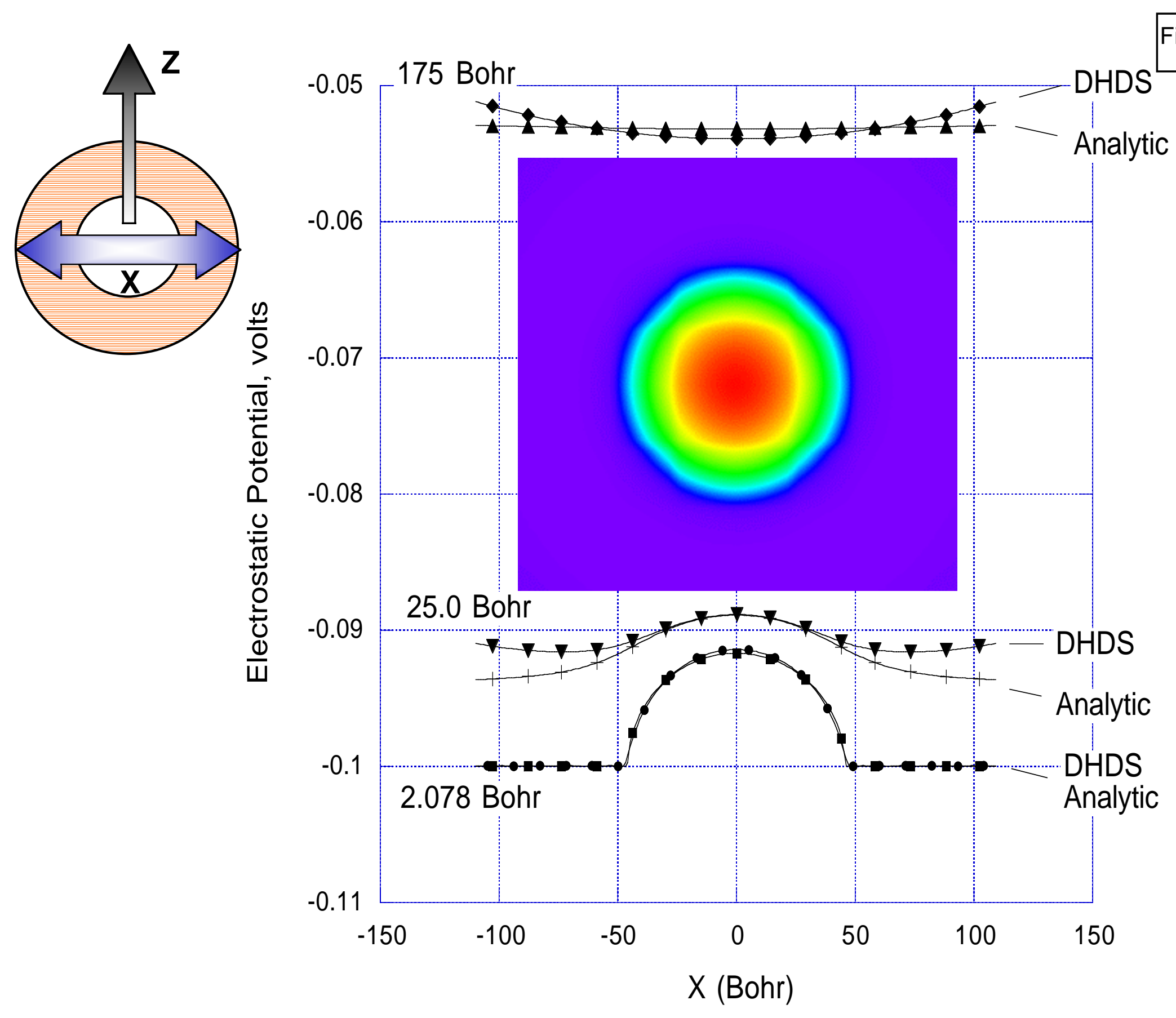



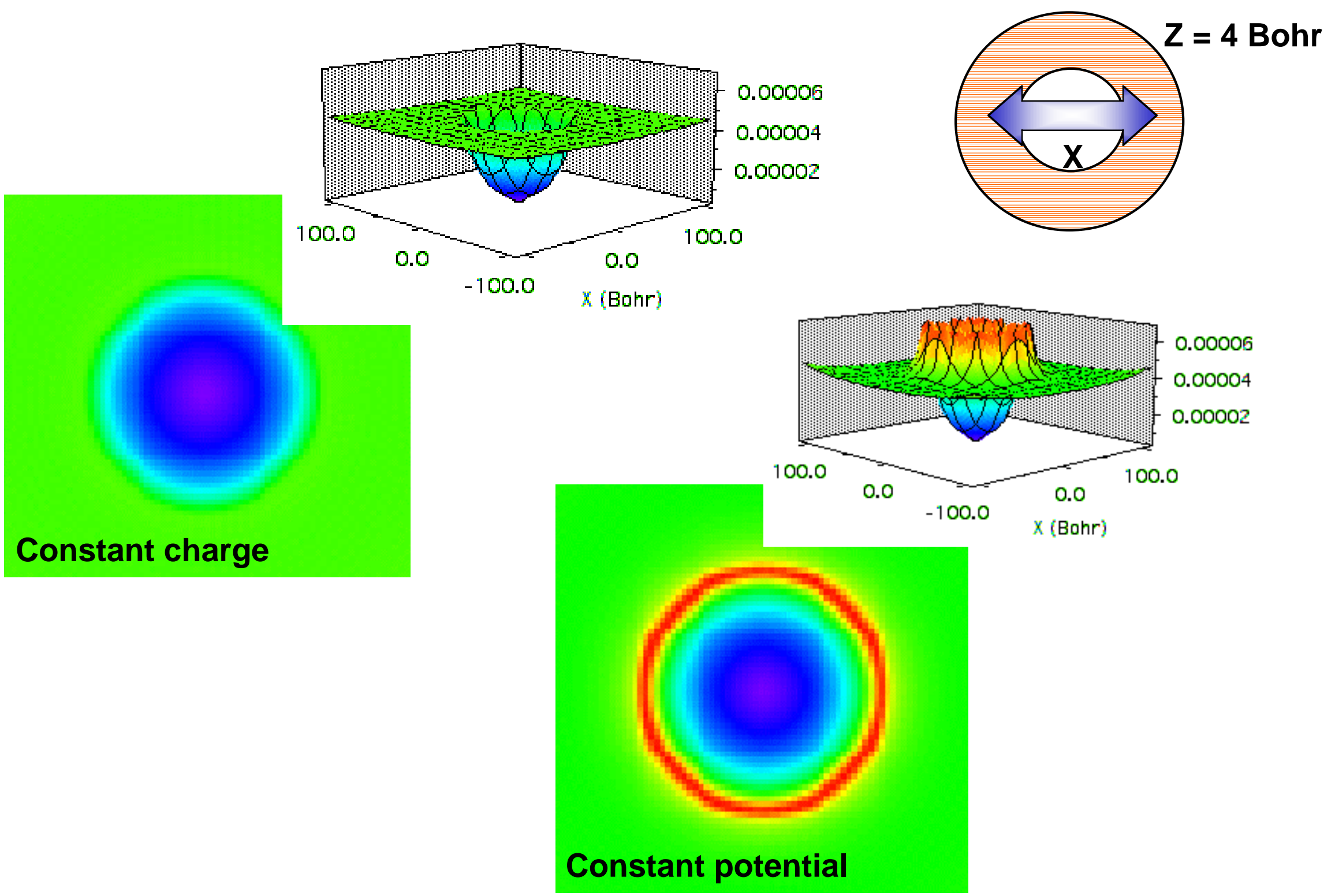
Fig. 8

Potential
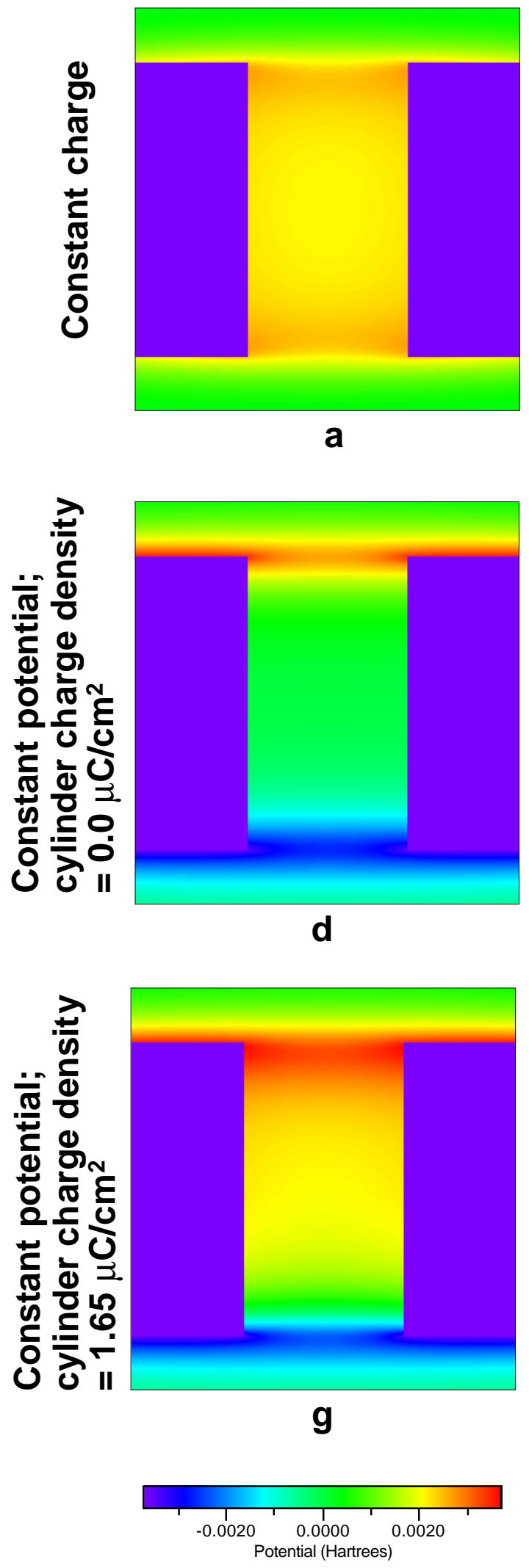

Positive ions

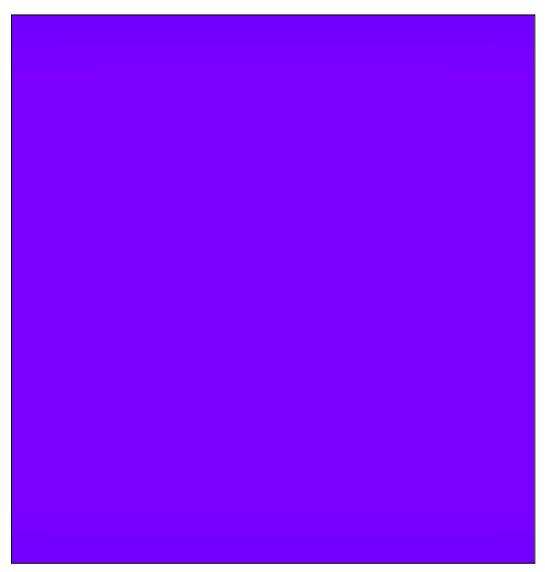

b

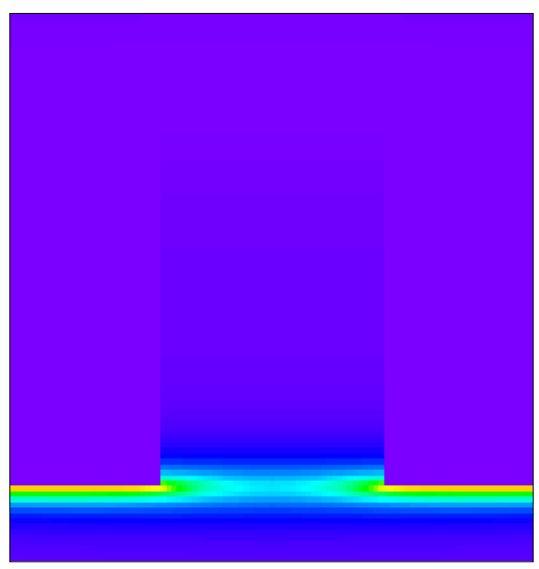

e

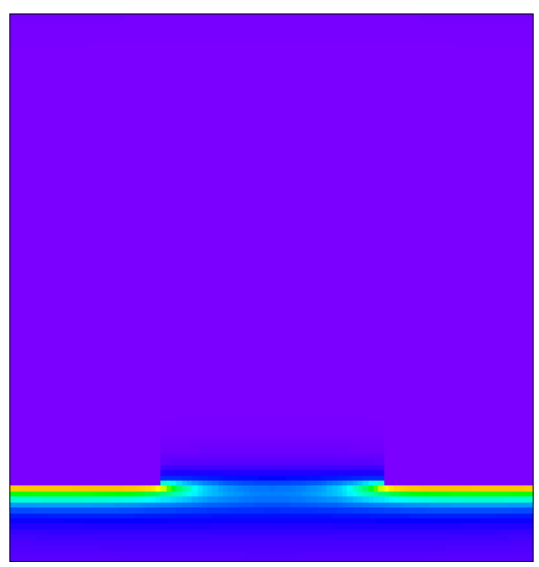

h

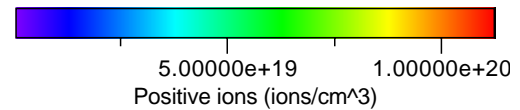

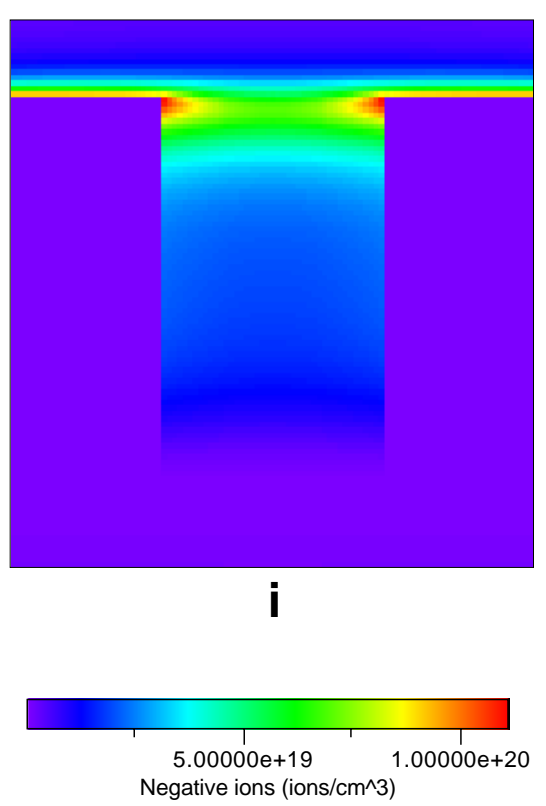

\section{Negative ions}

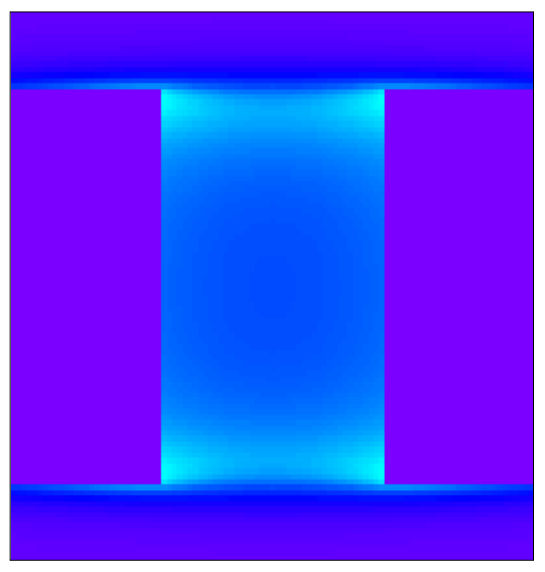

C

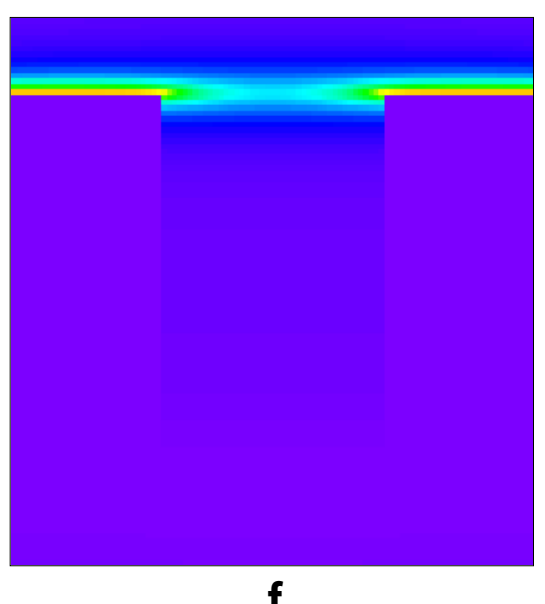

f 


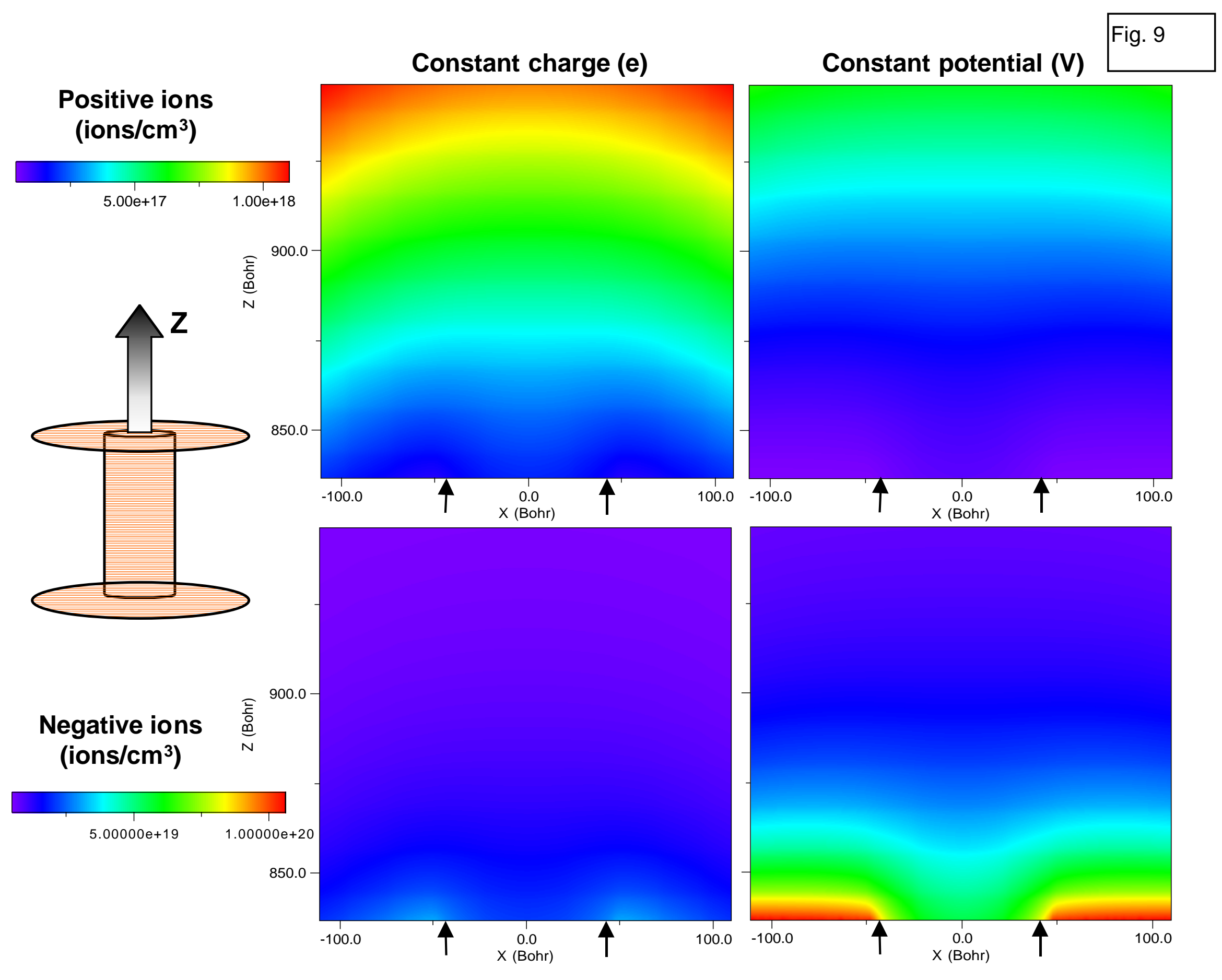

